\title{
Quantum information processing based on cavity QED with mesoscopic systems ${ }^{\star}$
}

\author{
Mikhail Lukin ${ }^{1}$, Michael Fleischhauer ${ }^{2}$, and Atac Imamoğlu ${ }^{3}$ \\ ${ }^{1}$ ITAMP, Harvard-Smithsonian Center for Astrophysics, Cambridge, MA 02138 \\ ${ }^{2}$ Sektion Physik, Universität München, D-80333 München, Germany \\ ${ }^{3}$ Dept. of Electr. \& Comp. Eng., Univ. of California, Santa Barbara, CA 93106
}

\section{Introduction}

Recent developments in quantum communication and computing [1-3] stimulated an intensive search for physical systems that can be used for coherent processing of quantum information. It is generally believed that quantum entanglement of distinguishable quantum bits (qubits) is at the heart of quantum information processing. Significant efforts have been directed towards the design of elementary logic gates, which perform certain unitary processes on pairs of qubits. These gates must be capable of generating specific, in general entangled, superpositions of the two qubits and thus require a strong qubit-qubit interaction. Using a sequence of single and two-bit operations, an arbitrary quantum computation can be performed [2].

Over the past few years many systems have been identified for potential implementations of logic gates and several interesting experiments have been performed. Proposals for strong qubit-qubit interaction involve e.g. the vibrational coupling of cooled trapped ions [4], near dipole-dipole or spin-spin interactions such as in nuclear magnetic resonance [5], collisional interactions of confined cooled atoms [6] or radiative interactions between atoms in cavity QED [7]. The possibility of simple preparation and measurement of qubit states as well as their relative insensitivity to a thermal environment makes the latter schemes particularly interesting for quantum information processing.

Most theoretical proposals on cavity-QED systems focus on fundamental systems involving a small number of atoms and few photons. These systems are sufficiently simple to allow for a first-principle description. Their experimental implementation is however quite challenging. For example, extremely high-Q micro-cavities are needed to preserve coherence during all atom-photon interactions. Furthermore, single atoms have to be confined inside the cavities for a sufficiently long time. This requires developments of novel cooling and trapping techniques, which is in itself a fascinating direction of current research. Despite these technical obstacles, a remarkable progress has been made in this area: quantum processors consisting of several coupled qubits now appear to be feasible.

\footnotetext{
* This work is dedicated to the memory of Professor Dan Walls
} 
On the other hand, some of the above difficulties are related to the microscopic nature of the system and may be avoided if mesoscopic systems are used. Proposals based on mesoscopic systems are also very attractive for possible large-scale implementation in the (presumably distant) future. Here collective (i.e. many-particle) excitations can be used as qubits, but it is in general difficult to control the coupling between them.

Motivated by this we here consider an approach that combines elements of cavity QED with mesoscopic systems. Specifically, we consider an $N$-atom system coupled to a few-photon cavity field. We investigate the conditions under which quantum entanglement can be created and manipulated in this mesoscopic system. Although entanglement manipulation involves collective rather than single-particle excitations, the system is still sufficiently simple to allow for a first principle description.

The central feature of our approach is the ability to manipulate collective excitations of light and matter by coherent control of the atom-field interaction using atomic dark resonances [8]. The present work demonstrates that the essential elements of QED-based quantum information processing can be implemented and that some of them can be considerably improved in a mesoscopic system. We show in particular that $(i)$ quantum information contained in polarization states of single photons can be stored in collective atomic excitations; ( $i i$ ) simple two-bit operations can be performed; (iii) entanglement can easily be transfered and distributed among collective excitations of distant atomic ensembles.

\section{Collective excitations as qubits}

A convenient way of encoding quantum information in optics is via the analogy between spin-1/2 systems and polarization states of light waves. We therefore begin by associating qubits with polarization states of single photons, and show that the states of these qubits can be mapped onto collective excitations of ensembles of atoms. We are here interested in single-photon excitations of cavity modes described by a superposition of right $\left(\left|1_{+}\right\rangle\right)$and left $\left(\left|1_{-}\right\rangle\right)$circularly polarized components

$$
\left|\Psi_{i}\right\rangle=\alpha_{i}\left|1_{i,+}\right\rangle+\beta_{i}\left|1_{i,-}\right\rangle,
$$

with $\left|\alpha_{i}\right|^{2}+\left|\beta_{i}\right|^{2}=1$. In the following we focus on the case that involves a pair of such single-photons states, i.e. $i=1,2$. For simplicity let us assume that the two photons occupy different frequency bands and hence are associated with different cavity modes.

In order to manipulate quantum information stored in such qubits we consider optical cavities filled with $N$ identical multilevel atoms. The frequency of a particular pair of transitions is assumed to be close to resonance frequencies of the cavity. The corresponding coupling strengths of the atoms 
to the two cavity modes $\hat{a}_{1+}$ and $\hat{a}_{1-}$ are assumed to be equal and are denoted by $g$ (see Fig.1a). In addition some time-dependent classical fields with Rabi-frequencies $\Omega_{1 \pm}(t)$ couple the lower (meta-stable) states $\left|c_{ \pm 1}\right\rangle$ of these atoms to the excited states $\left|a_{ \pm 1}\right\rangle$ as shown. The excited states decay with (equal) decay rate $\gamma$ and all atoms are initially prepared in a certain hyperfine sub-level, i.e. in a pure state.

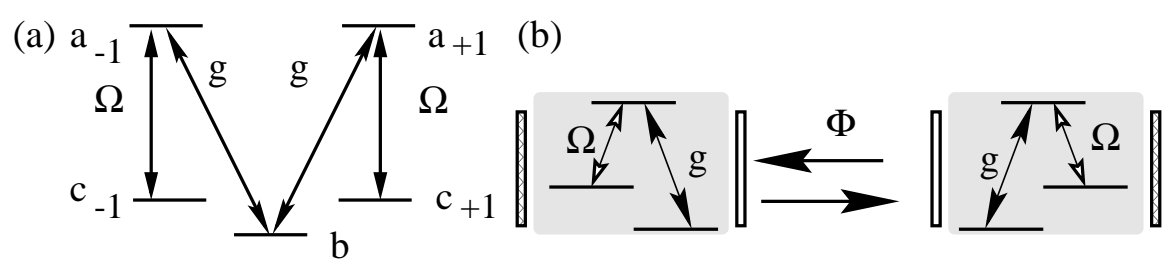

Fig. 1. (a) Schematic of the system for storing photon qubits in collective atomic excitations. (b) Quantum communication system based on photon trapping and release.

The basic Hamiltonian of the cavity + atom system can be written in terms of collective operators $\hat{\Sigma}_{a_{j}, b}=\sum_{i=1}^{N} \hat{\sigma}_{a_{j}, b}^{i}$ and $\hat{\Sigma}_{a_{j}, c_{j}}=\sum_{i=1}^{N} \hat{\sigma}_{a_{j}, c_{j}}^{i}$ as

$$
\hat{V}=\sum_{j} \hbar g \hat{a}_{j} \hat{\Sigma}_{a_{j}, b}+\hbar \Omega_{j}(t) \hat{\Sigma}_{a_{j}, c_{j}}+\text { h.c. }
$$

where $j=1 \pm$, and $\hat{\sigma}_{\mu \nu}^{i}=|\mu\rangle_{i i}\langle\nu|$ is the flip operator of the $i$ th atom between states $|\mu\rangle$ and $|\nu\rangle$. Here and below we work in a frame rotating with the optical frequencies.

Of special interest are certain superposition states of light and collective states of matter that do not interact with the optical fields. These socalled dark-states [8] correspond to elementary excitations of bosonic quasiparticles, so-called dark-state polaritons [9]. They are defined by the following canonical transformation

$$
\hat{d}_{j}=\cos \theta_{j} \hat{a}_{j}-\sin \theta_{j} \frac{1}{\sqrt{N}} \hat{\sigma}_{b c_{j}}, \quad \tan \theta_{j}(t)=g \sqrt{N} / \Omega_{j}(t)
$$

In the limit of small excitation the operators $\hat{d}_{j}$ and $\hat{d}_{j}^{\dagger}$ fulfill Bose commutation relations. The $\hat{d}_{j}^{\dagger}$ 's create a family of dark states which do not have an excited-state component and are decoupled from both optical fields:

$$
\left|D_{j i . .}, n_{j}, k_{i} \ldots\right\rangle=\frac{1}{\sqrt{n ! k ! \ldots}}\left(\hat{d}_{j}^{\dagger}\right)^{n}\left(\hat{d}_{i}^{\dagger}\right)^{k} \ldots|0\rangle|b\rangle_{1} \ldots|b\rangle_{N},
$$

$\hat{V}\left|D_{j i . .}, n_{j}, k_{i} \ldots\right\rangle=0$. These states are composed of cavity field states and symmetric Dicke-like atomic states $\left|\mathbf{c}_{j}^{n} \mathbf{c}_{i}^{k} ..\right\rangle$ containing $n$ atoms in level $\left|c_{j}\right\rangle$, 
$k$ atoms in level $\left|c_{i}\right\rangle$ etc, and all others in the ground state $|b\rangle$ :

$$
\begin{aligned}
|\mathbf{b}\rangle & \equiv|b\rangle_{1} \ldots|b\rangle_{N}, \quad\left|\mathbf{c}_{j}\right\rangle \equiv \sum_{l=1}^{N} \frac{-1}{\sqrt{N}}|b\rangle_{1} \ldots\left|c_{j}\right\rangle_{l} \ldots|b\rangle_{N}, \\
\left|\mathbf{c}_{j}^{2}\right\rangle & \equiv \sum_{l \neq m=1}^{N} \frac{1}{\sqrt{2 N(N-1)}}|b\rangle_{1} \ldots\left|c_{j}\right\rangle_{l} \ldots\left|c_{j}\right\rangle_{m} \ldots|b\rangle_{N}, \quad \text { etc. }
\end{aligned}
$$

We here assumed that the number of atoms is much larger than the number of photons in the light field.

The essence of the present approach is that a quantum bit stored in photo states can be transfered to collective atomic excitations (and vice versa) by adiabatic passage in dark-polariton states. Specifically single-mode dark states (4) have the following asymptotic behavior in the two limiting cases:

$$
\begin{gathered}
\left|D_{j}, n_{j}\right\rangle \rightarrow\left|n_{j}\right\rangle|\mathbf{b}\rangle, \quad \text { when } \quad \Omega \gg g \sqrt{N}, \\
\left|D_{j}, n_{j}\right\rangle \rightarrow|0\rangle\left|\mathbf{c}_{j}^{n}\right\rangle, \quad \text { when } \quad \Omega \ll g \sqrt{N} .
\end{gathered}
$$

It is most important that by varying the strength of the driving field $\Omega(t)$, the state of the combined atom+cavity system can be changed from cavitylike (in which excitation is mostly of photon nature) to atom-like (in which excitations are shared among the atoms). In the latter case the lifetime of excitations will not be sensitive to cavity decay; it will be limited solely by the decay of the meta-stable atomic states. In this process qubit states encoded in the photon field are mapped onto symmetric collective excitations of atomic ensembles. Since all dark states are orthogonal to each other, copying of all states can proceed in parallel.

It is known that adiabatic following takes place in the stimulated Raman process considered here, if the characteristic time scale $T$ exceeds the ratio of the optical decay rate $\gamma$ to the square of the characteristic Rabi-frequency. For the present system this condition translates into $g^{2} N / \gamma T \gg 1$. One recognizes that using a mesoscopic system with $N \gg 1$ considerably improves the adibaticity condition as compared to the single-atom case.

\section{Quantum entanglement of collective excitations}

A pair of qubits stored in collective excitations can be entangled using a number of different processes. Here we consider the resonantly enhanced Kerr effect [10] in combination with a cavity-QED setup to construct an elementary logic gate. The resonantly enhanced Kerr interaction in a 4-level configuration is the basis for the so-called "photon blockade" in a cavity configuration [11] and results in extremely strong photon-photon interactions of pulses [12].

To implement a two-bit gate we consider a pair of photons resonant with different transitions of the same multi-state atom. We use a level configuration and optical fields as indicated in Fig. 2. 

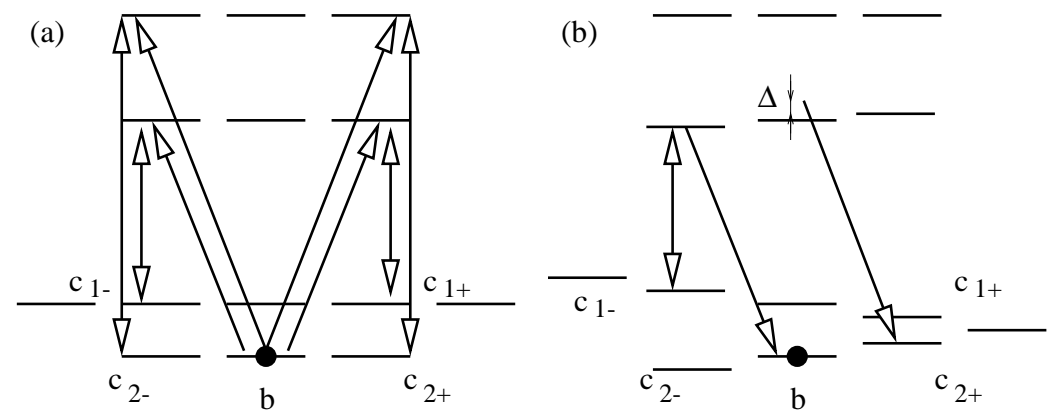

Fig. 2. Schematic of the system for an entanglement operation in atomic Rb. Only the coupling to the relevant transitions is shown.

In order to entangle qubit states the following sequence of operations can be used. In the first step [13], the photon state $\left|1_{1}\right\rangle=\alpha_{1}\left|1_{1+}\right\rangle+\beta_{1}\left|1_{1-}\right\rangle$ is transfered to collective atomic states composed of $\left|\mathbf{c}_{1 \pm}\right\rangle$ with the adiabatic technique described above. This operation corresponds to:

$$
\left(\alpha_{1}\left|1_{1+}\right\rangle+\beta_{1}\left|1_{1-}\right\rangle\right)|\mathbf{b}\rangle \rightarrow\left|0_{1}\right\rangle\left(\alpha_{1}\left|\mathbf{c}_{1+}\right\rangle+\beta_{1}\left|\mathbf{c}_{1-}\right\rangle\right) .
$$

In the next step, the state of the second photon $\left|1_{2}\right\rangle$ is mapped onto the different atomic sub-levels $\left|\mathbf{c}_{2 \pm}\right\rangle$ :

$$
\begin{aligned}
& \left(\alpha_{2}\left|1_{2+}\right\rangle+\beta_{2}\left|1_{2-}\right\rangle\right)\left(\alpha_{1}\left|\mathbf{c}_{1+}\right\rangle+\beta_{1}\left|\mathbf{c}_{1-}\right\rangle\right) \longrightarrow\left|0_{2}\right\rangle \times \\
& \left(\alpha_{1} \alpha_{2}\left|\mathbf{c}_{1+} \mathbf{c}_{2+}\right\rangle+\alpha_{1} \beta_{2}\left|\mathbf{c}_{1+} \mathbf{c}_{2-}\right\rangle+\beta_{1} \alpha_{2}\left|\mathbf{c}_{1-} \mathbf{c}_{2+}\right\rangle+\beta_{1} \beta_{2}\left|\mathbf{c}_{1-} \mathbf{c}_{2-}\right\rangle\right) .
\end{aligned}
$$

We now want to generate a conditional phase shift on only one of the collective states, say $\left|\mathbf{c}_{1-} \mathbf{c}_{2_{+}}\right\rangle$. For this we first apply a weak magnetic field in such a way that the transition $\left|\mathbf{c}_{1-}\right\rangle \rightarrow|\mathbf{b}\rangle$ becomes close to the frequency of some cavity mode (different from the one used for trapping of the photon $\left.\left|1_{1}\right\rangle\right)$. Note that this mode also couples off-resonantly (with detuning $\Delta$ ) the transition $\left|\mathbf{c}_{2+}\right\rangle \rightarrow\left|e_{2}, M_{F}=0\right\rangle$, where $|e\rangle$ denotes the excited state. The shift of the atomic energy levels will also result in undesired different phase shifts for the components of the collective atomic states. These phase shifts can be compensated however (e.g. by reversing the direction of the field for an appropriate time) and shall not be considered here.

By applying a classical field of appropriate frequency we can transfer one component of the collective state $\left|\mathbf{c}_{1-}\right\rangle$ back into the photonic mode:

$$
|0\rangle\left|\mathbf{c}_{1-} \mathbf{c}_{2+}\right\rangle \rightarrow\left|1_{1-}\right\rangle\left|\mathbf{c}_{2+}\right\rangle, \quad|0\rangle\left|\mathbf{c}_{1-} \mathbf{c}_{2-}\right\rangle \rightarrow\left|1_{1-}\right\rangle\left|\mathbf{c}_{2-}\right\rangle .
$$

At this point the energies of the states $\left|1_{1-}\right\rangle\left|\mathbf{c}_{2+}\right\rangle$ and $\left|1_{1_{-}}\right\rangle\left|\mathbf{c}_{2-}\right\rangle$ differ in a nontrivial way. Namely the state $\left|1_{1-}\right\rangle\left|\mathbf{c}_{2+}\right\rangle$ exhibits an AC-Stark shift $\delta=g^{2} / \Delta$, since it is coupled by the off-resonant cavity mode containing one photon. In order to avoid decoherence associated with two-photon absorption, 
$\Delta$ should exceed the optical decay rate $\gamma$. By simply letting the system evolve for a time $\tau$ a conditional phase $\Phi=\delta \tau$ is accumulated. By transferring the photonic components $\left|1_{1-}\right\rangle$ back to the atoms and reversing the magnetic field for a time appropriate to eliminate the single-bit phase shifts, the following state is obtained:

$$
\alpha_{1} \alpha_{2}\left|\mathbf{c}_{1+} \mathbf{c}_{2+}\right\rangle+\alpha_{1} \beta_{2}\left|\mathbf{c}_{1+} \mathbf{c}_{2-}\right\rangle+\mathrm{e}^{i \Phi} \beta_{1} \alpha_{2}\left|\mathbf{c}_{1-} \mathbf{c}_{2+}\right\rangle+\beta_{1} \beta_{2}\left|\mathbf{c}_{1-} \mathbf{c}_{2-}\right\rangle
$$

In the language of quantum information, this operation corresponds to a universal logic gate (a so-called phase gate) [1]. It is clear that by selecting a proper value of the conditional phase $\Phi$ and by performing independent single bit rotations, arbitrary entangled states of two qubits can be generated. This can be achieved however only if the system preserves coherence during the characteristic time required to accumulate a large phase shift. Hence, in the present approach $g^{2} \tau / \Delta \sim 1$ is required to achieve arbitrary entanglement of collective states. Thus while transfer operations as discussed in the previous section do not require a strong-coupling regime, two-bit operations still do.

\section{Effects of decoherence}

In this section we discuss the effect of decoherence on the manipulation of collective atomic excitations. In general, decoherence mechanisms depend on the particular implementation. In order to be specific we consider an ensemble of laser-cooled $\mathrm{Rb}$ atoms in a magneto-optic trap (MOT). The main sources of decoherence and dissipation are then $(i)$ spontaneous emission from the excited states (with the rate $\gamma$ ), (ii) the finite lifetimes of hyperfine and Zeeman coherences within the ground state (corresponding decay rate is $\gamma_{g}$ ) and, (iii) the photon decay of the optical cavity with rate $\gamma_{c}$.

For the present problem dephasing of the collective states is of interest. One finds that the states corresponding to single collective excitations are dephased at the same rate as the average coherences corresponding to individual atoms. For instance

$$
\frac{\mathrm{d}}{\mathrm{d} t}\left\langle\mathbf{b}|\rho| \mathbf{a}_{i}\right\rangle=\frac{\mathrm{d}}{\mathrm{d} t} \frac{1}{\sqrt{N}} \sum_{\mu=1}^{N}\left\langle\mathbf{b}|\rho| \mathbf{a}_{i}^{\mu}\right\rangle=-\gamma\left\langle\mathbf{b}|\rho| \mathbf{a}_{i}\right\rangle .
$$

By the same argument, coherences between hyperfine and Zeeman sub-levels decay at a rate $\gamma_{g}$. The states containing a single photon in a cavity mode will decay with an additional rate $\gamma_{c}$. In the following we assume that $\gamma_{g}$ is small on the time scales of interest and can be neglected.

Both processes considered in the previous sections are affected by decoherence, but in a different way. In the case of quantum state transfer, decoherence due to spontaneous emission can be avoided if the transfer time $T$ is sufficiently long such that the adiabatic following condition is fulfilled. However, in order to avoid decoherence due to cavity decay the transfer time $T$ should 
be short compared to $\gamma_{c}$. Hence, ideal quantum state transfer between cavity mode and collective excitations is only possible if

$$
g^{2} N \gg \gamma_{c} \gamma .
$$

In the case of two-bit operations, spontaneous emission causes two-photon absorption at a rate $\sim g^{2} / \Delta^{2} \gamma$. Here, two-photon absorption can be avoided when the detuning $\Delta$ (see Fig.2) is sufficiently large $\Delta \gg \gamma$. At the same time, the entanglement generation should be fast compared to the cavity decay $\tau \gamma_{c} \ll 1$. Hence, in order to accumulate a large conditional phase without dissipation it is necessary that

$$
g^{2} \gg \gamma_{c} \Delta \gg \gamma_{c} \gamma
$$

The main conclusion of this section is that in principle increasing the number of atoms does not make it harder to create quantum entanglement. Other operations such as the reliable quantum state transfer between light and matter become much easier. The reason for this behavior is that the basic decoherence mechanisms are not enhanced as the number of atoms is increased. At the same time the coupling of the cavity mode to the ground state is enhanced by a factor $\sqrt{N}$.

We note that in practice decoherence mechanisms exist that do scale with the number of atoms. For instance, off-resonant scattering of the external coherent fields on the transition from the ground $|b\rangle$ to the excited states $\left|e^{i}\right\rangle$ will result in dephasing of the collective states which is clearly enhanced: $\tilde{\gamma}=N \gamma \Omega^{2} / \tilde{\Delta}^{2}$. Here $\tilde{\Delta}$ is the (large) detuning of the coupling field from the $|b\rangle \rightarrow\left|e^{i}\right\rangle$ transition frequency. Therefore, in experiments extra care should be taken to avoid these decoherence mechanisms.

\section{Entanglement distribution}

One of the most intriguing aspects of quantum information is the use of entanglement as information resource for purposes such as super-dense information transfer [14], quantum teleportation [15] and secure communication [16]. In this section we show that the quantum state of collective atomic excitations including possible entanglements can be transferred form a given cavity system to other systems under much improved conditions as compared to single-atom QED systems. The technique is based on the possibility to map quantum correlations from traveling-wave light fields to collective atomic states and vice versa with nearly $100 \%$ efficiency $[17,18]$.

The basic mechanism is again the adiabatic procedure discussed in section 2 with the additional ingredient of a coupling to a continuum of free-space modes. We will outline the basic features for a single traveling-wave quantum field. In a suitable system, this operation can proceed in parallel for several field components and the corresponding generalization is straightforward. 
We consider a cavity with $N$ identical multi-level atoms as before. In addition we include the coupling of the cavity mode to a 1-D continuum of free-space modes with creation operators $b_{k}^{\dagger}$ described by the effective Hamiltonian $\hat{V}=\hbar \sum_{k} \kappa \hat{a}^{\dagger} \hat{b}_{k}+$ h.c.; $\kappa$ being the coupling constant. We assume that initially all atoms are in the ground state $|b\rangle$ and that there is no photon in the cavity. Thus the combined cavity-atom system is initially in the dark-state $|D, 0\rangle$ (see eq.(4)). The initial state of the free field is taken to be $\left|\Psi_{\text {in }}\right\rangle=\sum_{k} \xi_{k}^{1}\left|1_{k}\right\rangle+\sum_{k, m} \xi_{k, m}^{2}\left|1_{k} 1_{m}\right\rangle+\ldots$. It is convenient to work with correlation amplitudes, i.e. Fourier transforms of $\xi_{k \ldots l}^{j}$ :

$$
\Phi_{j}\left(t_{1} \ldots t_{j}\right)=\left\langle 0\left|\hat{E}\left(t_{1}\right) \ldots \hat{E}\left(t_{j}\right)\right| \Psi\right\rangle,
$$

where $\hat{E}(t)=L /(2 \pi c) \int d \omega_{k} \exp \left(i \omega_{k} t\right) \hat{b}_{k}$, and $L$ is the quantization length. E.g. $\Phi_{1}$ describes the envelope of a single-photon wave packet, $\Phi_{2}$ is the coincidence amplitude etc. We now consider a broad class of pulsed fields that are characterized by a single common envelope function $h(t)$ such that

$$
\Phi_{j}\left(t_{1}, t_{2}, \ldots t_{j}\right)=\alpha_{j} \sqrt{j !} h\left(t_{1}\right) h\left(t_{2}\right) \ldots h\left(t_{j}\right) .
$$

Any pure state or mixture of such pulses can be described by a single-mode density matrix $\rho_{n m}=\alpha_{n}^{*} \alpha_{m}$. The corresponding mode function is a superposition of plane waves proportional to $h(z / c)=\int \mathrm{d} \omega_{k} \xi_{k} \mathrm{e}^{i \omega_{k} z / c}$.

Due to the interaction of the cavity mode with the environment, the dark states of the cavity + atoms system are coupled to the continuum states. When only single-photon pulses are involved the evolution equations of the corresponding state amplitudes are [17]:

$$
\begin{aligned}
& \dot{D}_{1}(t)=i \kappa \cos \theta(t) \sum_{k} \xi_{k}(t), \\
& \dot{\xi}_{k}(t)=-i \Delta_{k} \xi_{k}(t)+i \kappa \cos \theta(t) D(t) .
\end{aligned}
$$

$D_{1}(t)$ denotes here the amplitude of the dark-state $|D, 1\rangle$ and $\theta(t) \equiv \theta_{1}(t)$ is defined in eq.(3). We proceed by formally integrating Eq.(19), substituting the result into Eq.(18) and invoking a Markov approximation. Assuming that no photons arrive to the cavity before $t_{0}$ we find for the dark state amplitude $D_{1}(t)=-i \alpha_{1} D(t)$ with

$$
D(t)=\sqrt{\gamma_{c} \frac{c}{L}} \int_{t_{0}}^{t} \mathrm{~d} \tau \cos \theta(\tau) h(\tau) \times \exp \left\{-\frac{\gamma_{c}}{2} \int_{\tau}^{t} \mathrm{~d} \tau^{\prime} \cos ^{2} \theta\left(\tau^{\prime}\right)\right\} .
$$

Here we have introduced the empty-cavity decay rate $\gamma_{c}=\kappa^{2} L / c$. Substituting this result back into eq.(19) one finds that the outgoing field is described by the common envelope function $h_{\text {out }}(t)=h(t)-\sqrt{\gamma_{c} L / c} D(t)$.

In order to trap photons we require that the envelope of the outgoing field and its first derivative vanish identically. I.e. $h_{\text {out }}(t)=\dot{h}_{\text {out }}(t)=0$. Differentiating the above relation for $h_{\text {out }}(t)$ yields

$$
-\frac{\mathrm{d}}{\mathrm{d} t} \ln \cos \theta(t)+\frac{\mathrm{d}}{\mathrm{d} t} \ln h(t)=\frac{\gamma_{c}}{2} \cos ^{2} \theta(t) .
$$


If $\Omega(t)$ is chosen such that $\theta(t)$ obeys this equation with the asymptotic condition $\cos \theta \rightarrow 0$ the output field remains zero and the incoming light pulse is completely transferred to the atomic system.

The above condition corresponds to a quantum or dynamical impedance matching [17]. The term on the r.h.s. of Eq.(21) is the effective cavity decay rate reduced due to intracavity electromagnetically induced transparency (EIT) [19]. The first term on the l.h.s. describes internal "losses" due to coherent Raman adiabatic passage and the second term is due to the timedependence of the input field. As in the case of classical impedance matching [20], Eq. (21) reflects the condition for complete destructive interference resulting in a vanishing outgoing wave. Solving Eq.(21) yields

$$
\cos ^{2} \theta(t)=\frac{h^{2}(t)}{\gamma_{c} \int_{-\infty}^{t} \mathrm{~d} \tau h^{2}(\tau)},
$$

which corresponds to $D(t \rightarrow+\infty) \rightarrow 1$. Hence, by suitable variation of the classical driving field any single-photon pulse can be trapped ideally, if its pulse length is longer than the bare-cavity decay time.

Generalizations of the above considerations to multi-photon states can proceed along the same lines, but involve more tedious algebra. In particular, for the two-photon states one finds $D_{2}(t)=-\alpha_{2} D(t)^{2}$, and in general

$$
D_{k}(t)=(-i)^{k} \alpha_{k} d(t)^{k}
$$

can be proved. Under conditions of quantum impedance matching $D_{k}(t \rightarrow$ $\infty) \rightarrow(-i)^{k} \alpha_{k}$ for arbitrary $k$. Hence pulsed fields in a generalized single mode with arbitrary quantum state can be mapped onto the atomic ensemble.

Releasing the stored quantum state into a pulse of desired shape can be accomplished in a straightforward way. A simple reversal of the time dependence of the control field at a later time $t_{d}$ leads to a perfect mirror-image of the initial pulse. This can be verified directly from Eqs. (20).

Before concluding we note that the quantum transfer protocol described here is based solely on the adiabatic rotation of the dark state described in Section 2. Hence, this operation can be nearly ideal whenever inequality (14) is fulfilled [17]. Therefore, perfect quantum communication can be achieved in the present system without invoking the strong coupling regime of cavity QED.

\section{Conclusions}

In conclusion, we have shown that quantum information stored in collective excitations of an $\mathrm{N}$-atom system and can be coherently processed using cavity QED techniques. We showed that certain network operations such as the transfer of excitation between atomic and photonic degrees of freedom and entanglement distribution can be performed without invoking the strong 
coupling condition of cavity QED. However other operations, such as two-bit rotations resulting in quantum entanglement still require a strong coupling. Studies of possible ways to alleviate these requirements, and to avoid the strong coupling regime altogether are currently under way. This includes, for instance, resonant nonlinearities in a traveling wave geometry, so-called photon-exchange interactions or cold collisions.

This work was supported by the National Science Foundation via the grant to the Institute for Theoretical Atomic and Molecular Physics. We thank Susanne Yelin for stimulating discussions and collaboration on a related project. One of us (ML) especially thanks her and little Theodor for their patience that allowed to complete this contribution.

\section{References}

1. R. P. Feynman, Int. J. Theor. Phys. 21, 467 (1982); D. Deutsch, Proc. R. Soc. London A 425, 73 (1989).

2. see e.g.: D. P. DiVincenzo, Science 270, 255 (1995); C. H. Bennett, Phys. Today 48, No. 10, 24 (1995); A. Ekert and R. Josza, Rev. Mod. Phys. 68, 733 (1996); A. Steane Rep. Prog. Phys. — bf 61, 117 (1998).

3. D. Deutsch, Proc. R. Soc. London A 400, 97 (1985).

4. J. I. Cirac and P. Zoller, Phys. Rev. Lett. 74, 4091 (1995).

5. N. A. Gershenfeld and I. L. Chuang, Science 275, 350 (1997).

6. D. Jaksch et al., Phys. Rev. Lett. 82, 1975 (1999).

7. T. Pellizzari et al., Phys. Rev. Lett. 75, 3788 (1995).

8. see e.g.: E. Arimondo, Progr. in Optics 35, 259 (1996);

9. M. Fleischhauer and M. D. Lukin, (preprint quant-ph/0001094)

10. H. Schmidt and A. Imamoğlu, Opt. Lett. 21, 1936 (1996).

11. A. Imamoğlu et al., Phys. Rev. Lett. 79, 1467 (1997); P. Grangier, D. F. Walls, and K. Gheri, Phys. Rev. Lett. 81, 2833 (1998).

12. M. D. Lukin and A. Imamoğlu, Phys. Rev. Lett. 84, 1419 (2000).

13. Note that the order in which the qubits are trapped is in principle important. Corresponding correction can be disregarded however when $N \gg 1$.

14. C. H. Bennet and S. J. Wiesner, Phys. Rev. Lett. 69, 2881 (1992); K. Mattle et al., Phys. Rev. Lett. 76, 4656 (1996).

15. C. H. Bennett et al., Phys. Rev. Lett. 70, 1895 (1990); B. Bouwmeester et al., Nature 390, 575 (1997); D. Boschi et al., Phys. Rev. Lett. 80, 1121 (1998); A. Furusawa et al. Science 282, 706 (1998).

16. C. H. Bennett and G. Brassard, Proc. of IEEE Int. Conf. on Comp. Systems and Signal Processing, Banglore India (IEEE, New York 1984); A. K. Ekert, Phys. Rev. Lett. 67, 661 (1991).

17. M. Fleischhauer, S. F. Yelin, and M. D. Lukin, Opt. Comm. (2000) in press

18. M. D. Lukin, S. F. Yelin, and M. Fleischhauer, Phys. Rev. Lett. (2000) (preprint quant-ph/9912046).

19. M. D. Lukin et al., Opt. Lett. 23, 295 (1998).

20. A. Siegmann, Lasers, (University Science Books, Mill Valley CA, 1986). 